\title{
Fondsgebundene Lebensversicherung mit Höchststandgarantie - der neue Trend
}

\author{
Roger Ledermann
}

Korrespondenz:

Roger Ledermann

FMH Insurance \& Financial

Services AG

Moosstrasse 2

CH-3073 Gümligen

Tel. 0319595000

Fax 0319595010

E-Mail: info@fmhinsurance.ch
Vor rund zehn Jahren bewegte der aufkommende Fondstrend die Versicherungsgesellschaften zur Lancierung eines neuen Produktes: die fondsgebundene Lebensversicherung. Die Nachfrage nach diesem Produkt war derart stark, dass bereits wenige Jahre später jeder zweite Sparfranken in eine fondsgebundene Versicherung floss.

Angesichts der Kursverluste in den vergangenen Jahren und der heute teilweise stark schwankenden Aktienmärkte erkennen wir einen Wandel in den Bedürfnissen der Anleger. Heute ist die Sicherheit des Kapitals für viele Investoren das oberste Ziel. Dennoch möchten sie von den Chancen der Kapitalmärkte profitieren können.

Auch einige Versicherungsgesellschaften haben diese Bedürfnisse erkannt und bieten heute das Produkt «fondsgebundene Lebensversicherung mit Kapitalgarantie» an. Bei dieser Anlageform erhalten die Investoren auch bei einem vermeintlichen Verlust ihrer Anlage eine Mindestsumme garantiert ausbezahlt. Musste man sich bisher eine solche Garantie teuer erkaufen und auf einen grossen Teil der Gewinnchancen verzichten, verfügen die heutigen Fonds über eine dynamische Anlagestrategie, welche sich laufend den aktuellen Marktverhältnissen anpasst.

Dadurch ist es nun auch möglich, bereits erreichte Gewinne auf das Ende der Laufzeit ab- zusichern. Bei den sogenannten «Fonds mit Höchststandgarantie» erhält der Anleger den höchsten je erreichten Wert seiner Anlage, und zwar unabhängig davon, wann und zu welchem Kurs er seine Anteile gekauft hat.

Erwirbt man diese Anlage in Kombination mit einer Lebensversicherung, profitiert man von folgenden zusätzlichen Vorteilen:

- steuerfreie Auszahlung (unter gewissen Voraussetzungen);

- zusätzlicher Versicherungsschutz im Todesfall;

- Möglichkeit, das Sparziel mit dem Einschluss einer Prämienbefreiung bei Erwerbsunfähigkeit abzusichern;

- erbrechtliche Vorteile;

- Konkursprivileg.

Diese Produkte sind oftmals so konzipiert, dass sie sich sowohl für die freie Vorsorge (Säule 3b) wie auch für die gebundene Vorsorge (Säule 3a) einsetzen lassen. Zudem ist die Finanzierung mit einer periodischen oder mit einer einmaligen Zahlung möglich.

Die Angebote der Gesellschaften unterscheiden sich je nach Ausgangslage stark und werden laufend angepasst. Nur durch das Einholen und Vergleichen mehrerer Offerten kann der am besten geeignete Anbieter gefunden werden.

\begin{tabular}{|c|c|c|c|c|c|}
\hline Versicherte Person & \multicolumn{5}{|c|}{ Mann, 1.4.1960 } \\
\hline Jahresprämie & \multicolumn{5}{|l|}{ Fr. $10000 .-$} \\
\hline Versicherungsdauer & \multicolumn{5}{|l|}{20 Jahre } \\
\hline Beginn & \multicolumn{5}{|l|}{1.4 .2005} \\
\hline \multirow[t]{2}{*}{ Angenommene Fondsrendite } & \multicolumn{5}{|l|}{$4 \%$} \\
\hline & Angebot 1 & Angebot 2 & Angebot 3 & Angebot 4 & Angebot 5 \\
\hline Todesfallsumme & 201566 & 197239 & 200000 & 214083 & 201207 \\
\hline Wert der Fondsanteile & 266400 & 264481 & 264144 & 263810 & 250130 \\
\hline Rendite & $2,66 \%$ & $2,59 \%$ & $2,58 \%$ & $2,57 \%$ & $2,08 \%$ \\
\hline
\end{tabular}

